\title{
A High Granularity Timing Detector for the Phase-2 Upgrade of the ATLAS Experiment: Detector concept, $R \& D$, and first test beam results
}

\author{
Ariel Schwartzman, on behalf of the ATLAS Collaboration* \\ SLAC National Accelerator Laboratory \\ E-mail: scheslac.stanford.edu
}

The expected increase of the particle flux at the high luminosity phase of the LHC (HL-LHC) with instantaneous luminosities up to $L=7.5 \times 10^{34} \mathrm{~cm}^{-2} \mathrm{~s}^{-1}$ will have a severe impact on the ATLAS detector performance. The pile-up is expected to increase on average to 200 interactions per bunch crossing. The reconstruction and trigger performance for electrons, photons as well as jets and transverse missing energy will be severely degraded in the end-cap and forward region, where the Liquid Argon based electromagnetic calorimeter has coarser granularity and the inner tracker has poorer momentum resolution compared to the central region. A High Granularity Timing Detector (HGTD) is proposed in front of the Liquid Argon end-cap calorimeters for pileup mitigation and for bunch per bunch luminosity measurements. This device should cover the pseudo-rapidity range of 2.4 to about 4.0. Two Silicon sensors double sided layers are foreseen to provide a precision timing information for minimum ionizing particles with a time resolution better than 50 pico-seconds per hit (i.e 30 pico-seconds per track) in order to assign the particle to the correct vertex. Each readout cell has a transverse size of $1.3 \mathrm{~mm} \times 1.3 \mathrm{~mm}$ leading to a highly granular detector with about 3 millions of readout electronics channels. Low Gain Avalanche Detectors (LGAD) technology has been chosen as it provides an internal gain good enough to reach large signal over noise ratio needed for excellent time resolution. The requirements and overall specifications of the High Granular Timing Detector at the HL-LHC will be presented as well as the conceptual design. Most recent results on the main R\&D will be discussed, with emphasis on the LGAD sensors (sensor optimisation as thickness, dead zone, and radiation hardness) and ASIC. Beam test results of gain, timing resolution and efficiency will be also shown.

The 39th International Conference on High Energy Physics (ICHEP2018)

4-11 July, 2018

Seoul, Korea

${ }^{*}$ Speaker. 


\section{Physics motivation}

Pileup is one of the most difficult challenges at the HL-LHC. It creates additional jets that need to be suppressed, and reduces the precision of physics objects. One of the key elements to mitigate the effect of pileup on physics event reconstruction is the precise assignment of tracks to primary vertices, which allows to discriminate between charged particles originating from the hard-scatter vertex and pileup interactions. Track-to-vertex association, however, becomes extremely challenging particularly in the forward region, where the track longitudinal impact parameter resolution may be larger than the separation between nearby primary vertices. In this case, the association of tracks to primary vertices becomes ambiguous as a same track may be compatible with multiple nearby vertices, reducing the power of pileup mitigation techniques for jets, $b$-tagging, lepton isolation, and missing transverse energy. For example, for a local pileup density of 1.5 collisions $/ \mathrm{mm}$ corresponding to 200 pileup interactions, the typical average separation between primary vertices at $z=0$ will be approximately $0.6 \mathrm{~mm}$. This has to be compared with a track $z$-impact parameter resolution of $4 \mathrm{~mm}$ for tracks with $p_{\mathrm{T}}=1 \mathrm{GeV}$ and $|\eta|>3$. A powerful new way to address this challenge is to exploit the spread in time of the collisions within each bunch crossing to distinguish between vertices occurring very close in space but well separated in time. This requires the ability to measure the time of individual tracks with a precision smaller than the time spread of collisions. In particular, for a nominal time spread of $180 \mathrm{ps}$, a timing detector with a resolution of $30 \mathrm{ps}$ per track would reduce the amount of pileup by a factor of 6 , bringing it to levels comparable to Run 2 .

\section{HGTD detector concept}

The High Granularity Timing Detector (HGTD) will consist of two endcap disks covering the pseudorapidity region $2.4<|\eta|<4$.0. Each disk will be located at $z=3.5 \mathrm{~m}$ with a radial extension (R) between $12 \mathrm{~cm}$ and $64 \mathrm{~cm}$. Each endcap disk will contain two double planar layers to provide an average number of HGTD hits per track between 2 and 3 depending on the distance from the beam axis.

The key HGTD requirements are a time resolution of 30 ps per track, flat in $\eta$, radiation tolerance up to $3.7 \times 10^{15} \mathrm{n}_{\mathrm{eq}} / \mathrm{cm}^{2}$ and $4.1 \mathrm{MGy}$, low electronic noise, and low occupancy. These requirements can be achieved using Low Gain Avalanche Detector sensors (LGADs) with a pixel size of $1.3 \times 1.3 \mathrm{~mm}^{2}$. The choice of two layers will ensure a per-track time resolution of $30 \mathrm{ps}$ after electronic noise and sensor per-hit time resolution degradation due to radiation are taken into account. Since the LGAD hit time resolution worsens with smaller radial distance due to larger radiation dose, the HGTD is designed to increase the number of hits at low radius. This is achieved with a larger overlap (80\%) of modules for $R<32 \mathrm{~cm}(|\eta|>3.1)$, compared to $20 \%$ for larger R. Furthermore, to ensure a track time resolution of $30 \mathrm{ps}$ at the end of the lifetime, it is planned to replace the inner part of the HGTD after half of the HL-LHC program.

The main contributions to the time resolution of a silicon sensor and its associated readout electronics are given by

$$
\sigma_{t}^{2}=\sigma_{\text {Landau }}^{2}+\sigma_{\text {time-walk }}^{2}+\sigma_{\text {jitter }}^{2}+\sigma_{T D C}^{2}+\sigma_{\text {clock }}^{2}
$$

The first term corresponds to the event-by-event Landau fluctuations of the charge deposition within the sensor. Landau fluctuations set the ultimate (physics) limit to the time resolution. The time- 
walk and jitter components are proportional to the rise time of the signal and inversely proportional to the signal over noise ratio $(\mathrm{S} / \mathrm{N})$. The fourth term is given by the resolution of the time-to-digital converter (TDC). For a 20 ps TDC, this resolution is $20 \mathrm{ps} / \sqrt{12} \sim 5 \mathrm{ps}$. Based on expression 2.1, the key to precision timing consists of a large signal with short rise time and low noise, which can be achieved with the use of LGAD sensors. LGADs, shown schematically in Figure 2, are n-on-p silicon detectors with gain from an additional highly doped p-layer that creates a high electric field. LGAD sensors are thin, resulting in a small signal rise time, provide a moderate gain to increase the signal while limiting the noise at the same time, and are radiation hard.

The single pixel readout electronics consists of a preamplifier, followed by a discriminator and two TDCs. One TDC is used to measure the time of arrival (TOA), and another provides time-over-threshold (TOT) information for time-walk corrections. The TOA TDC is a 7 bit high precision 20 ps TDC, with a range of $2.5 \mathrm{~ns}$. The 9 bit TOT TDC uses the same TDC architecture but has a coarser binning of $40 \mathrm{ps}$ and expanded range of $20 \mathrm{~ns}$. The TDC design uses a Vernier configuration with two delay lines of 120 ps and 140 ps as shown schematically in Figure 2. The TDC time resolution in this Vernier configuration is given by the difference in delay of the cells in each line. When a trigger signal is received, the start signal is sent to the START delay line, where each cell has a delay of $140 \mathrm{ps}$. The detection of a signal hit in a pixel sends the stop signal to the faster (120 ps) STOP delay line. As the signals move through the delay cells, the STOP signal will eventually reach and pass the START signal. The TOA is therefore determined by the number of stages needed for the STOP signal to surpass the START signal.

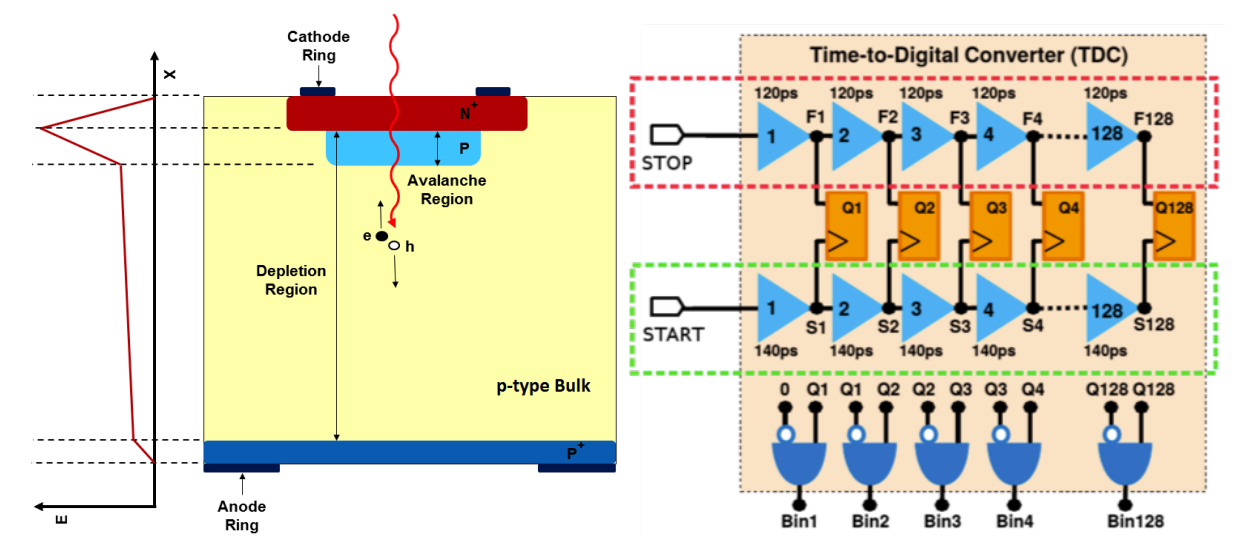

Figure 1: Schematic of a LGAD sensor (Left) and the Time-to-Digital Converter (Right) [2].

\section{Test beam results}

Five different periods of beam tests during 2016 and 2017 took place using the H6 beam line of the CERN SPS with $120 \mathrm{GeV}$ pions [3]. Time resolutions are extracted from the spread in the difference between the time-of-arrival of two sensors when a particle passes through both. For details on the beam test configuration and measurements see [3].

Figure 3 shows time resolution results for various types of single pad sensors and $2 \times 2$ arrays produced by CNM. For un-irradiated sensors, shown on the left panel, an ultimate time resolution of $30 \mathrm{ps}$, limited by the Landau, term is observed. Devices with a high doping dose show worse 
time resolution at a fixed gain due to the lower applied voltage required for their operation. Lower voltage directly translates into larger rise times. The main effect of radiation is to reduce doping concentration leading to a decrease in the sensor gain. The right panel of Figure 3 shows the fluence dependence of the time resolution for HPK LGAD sensors of 35 and $50 \mu m$ thickness (B35 and 50D). A time resolution smaller than $50 \mathrm{ps}$ up to $5 \times 10^{15} \mathrm{n}_{\mathrm{eq}} / \mathrm{cm}^{2}$ with bias voltage (VHR) $10 \%$ below breakdown voltage (VBD) is achieved. Sensors with $35 \mu \mathrm{m}$ thickness show similar time resolution to those with $50 \mu \mathrm{m}$ at high fluence, but better below $1 \times 10^{15} \mathrm{n}_{\mathrm{eq}} / \mathrm{cm}^{2}$. The signal efficiency is found to be larger than $99.8 \%$ and the time resolution is uniform and within 30 ps to $40 \mathrm{ps.}$
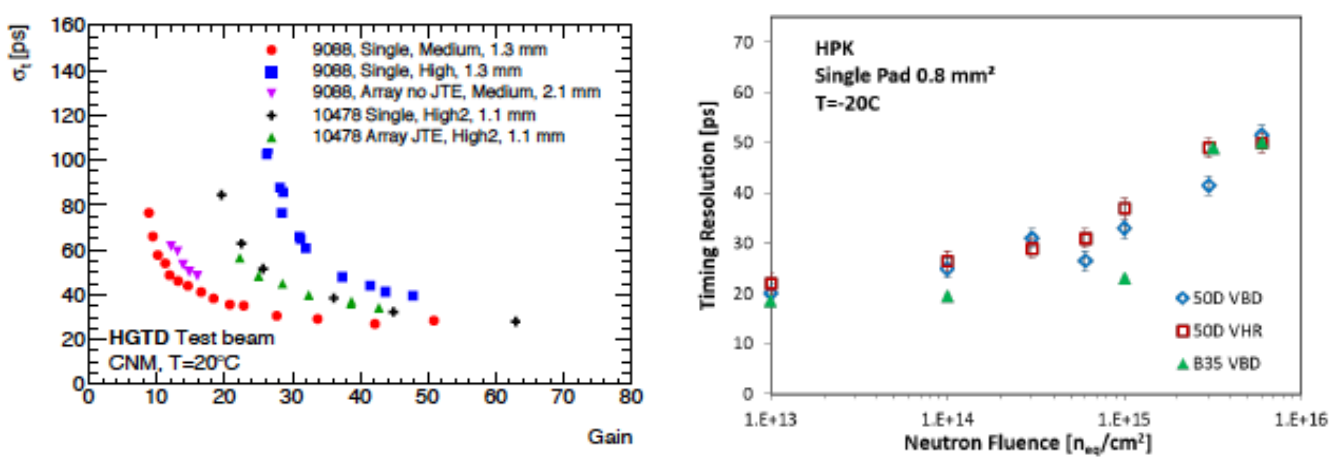

Figure 2: Time resolution as a function of gain (Left), and neutron fluence (Right) [3].

\section{Summary}

For the first time, HL-LHC will operate a forward tracker in an environment in which the pileup density is higher than its $z$ spatial resolution. The use of precise timing information will enable the accurate assignment of low $p_{\mathrm{T}}$ forward tracks to vertices, enhancing the capabilities of the inner tracker to suppress pileup. HGTD brings an entirely new capability in hadron colliders that will enhance the physics potential of the ATLAS detector, particularly in Vector Boson Fusion and Vector Boson Scattering topologies, and in final states containing forward $b$-jets and leptons. ATLAS has an extensive R\&D program to test LGAD ultra-fast silicon sensors and develop dedicated ASIC readout electronics. The HGTD technical proposal was approved in June 2018 and the Technical Design Report is planned for the end of March 2019.

\section{References}

[1] ATLAS Collaboration, The ATLAS Experiment at the CERN Large Hadron Collider, 2008 JINST 3 S08003.

[2] ATLAS Collaboration, Technical Proposal: A High-Granularity Timing Detector for the ATLAS Phase-II Upgrade, CERN-LHCC-2018-023; LHCC-P-012.

[3] C. Allaire et al., Beam test measurements of Low Gain Avalanche Detector single pads and arrays for the ATLAS High Granularity Timing Detector, JINST 13 P06017 (2018), arXiv:1804.00622 [physics.ins-det]. 